\title{
Pelvic support osteotomy by Ilizarov's concept: Is it a valuable option in managing neglected hip problems in adolescents and young adults?
}

\author{
Mahmoud A. Mahran • Mohamed A. ElGebeily • \\ Nabil A. M. Ghaly • Mootaz F. Thakeb • \\ Hany M. Hefny
}

Received: 29 March 2009/ Accepted: 13 January 2011/Published online: 1 March 2011

(c) The Author(s) 2011. This article is published with open access at Springerlink.com

\begin{abstract}
For evaluating pelvic support osteotomy as a salvage procedure in managing neglected hip problems in adolescents and young adults, PSO was performed for 20 hips in 20 patients ( 5 men and 15 women). The mean age was 21.5 years. The etiology was neglected developmental dysplasia of the hip in 9 patients, post-septic hip sequelae in 9 patients, and paralytic dislocation due to poliomyelitis in 2 patients. All patients were treated by two osteotomies: a proximal femoral osteotomy to support the pelvis and correct the flexion and rotational deformities of the hip and a distal varization and lengthening osteotomy. Final clinical evaluation was done 6 months after frame removal. The mean external fixation time was 6.4. Lengthening and mechanical axis parallelism was achieved in all patients. At the final follow-up and according to a predesigned scoring system, there were $7(35 \%)$ excellent results, $6(30 \%)$ good results, $7(35 \%)$ fair results, and no poor results. Hip reconstruction by Ilizarov's concept can be technically demanding and involving lengthy period wearing the frame but found to be a valuable salvage procedure for numerous neglected hip problems particularly in young patients.
\end{abstract}

Keywords Pelvic support osteotomy - Hip osteotomy · Femoral reconstruction osteotomy $\cdot$ Neglected hip dislocation - Pelvic support femoral reconstruction . Neglected hip problems in pediatric age group . Ilizarov hip reconstruction

\footnotetext{
M. A. Mahran $(\varangle) \cdot$ M. A. ElGebeily ·

N. A. M. Ghaly - M. F. Thakeb - H. M. Hefny

Orthopedic department, Ain Shams

University Medical School and Hospitals, Cairo, Egypt

e-mail: mahmoudmahran@gmail.com

M. F. Thakeb

e-mail: mootaz_thakeb@yahoo.com
}

\section{Introduction}

Instability of the hip in teenagers is a difficult problem to treat. Usually, shortening and femoral bone loss complicate the problem. For patients with unilateral hip pathology, arthrodesis can be a satisfactory salvage operation. However, loss of range of motion and ipsilateral hip and knee problems are the main consequences [1-3]. With advances in metallurgy, total hip replacement has become the first choice of treatment for patients with unstable hips. It gives patients painless hips with a good range of motion. However, in younger age group, these prostheses are subjected to substantial mechanical stresses and consequently early failures [3-5].

Osteotomies around the hip, whether deformity correcting or deformity producing, aim at reorienting biological tissues to improve gait mechanics. Pelvic support osteotomy (PSO) evolved to solve problems associated with hip instability by supporting the pelvis on the upper end of osteotomized femur [3, 6]. Bouvier, in 1838, first performed subtrochanteric osteotomy aiming at pelvic support, in congenital dislocation of the hip [7]. Kirmission, in 1894, suggested femoral osteotomy in the treatment of irreducible dislocation of long duration aiming to correct the frequently present adduction contracture $[8,9]$.

Von Baeyer, in 1918, made a subtrochanteric osteotomy aiming of increasing tension in the pelvifemoral muscles so that they could support the pelvis better (Fig. 1a, b) [4]. Adolf Lorenz devised his bifurcation osteotomy to correct deformity and to restore stability during weight bearing [10]. This was later modified by Schanz and Hass [9].

With the advent of hip arthroplasty, these osteotomies were forgotten. Till, G. A. Ilizarov, in the eighties, used his apparatus and the biologic principles that he elucidated to 
Fig. 1 Subtrochanteric femoral osteotomy provides a stable fulcrum (arrow in 1b) for pelvic support, increases abductor lever arm (narrower right-left arrow 1 in $1 \mathrm{~b}$ in comparison with arrow 2 in $2 b$ ), and retention the pelvifemoral muscles (hip abductors)
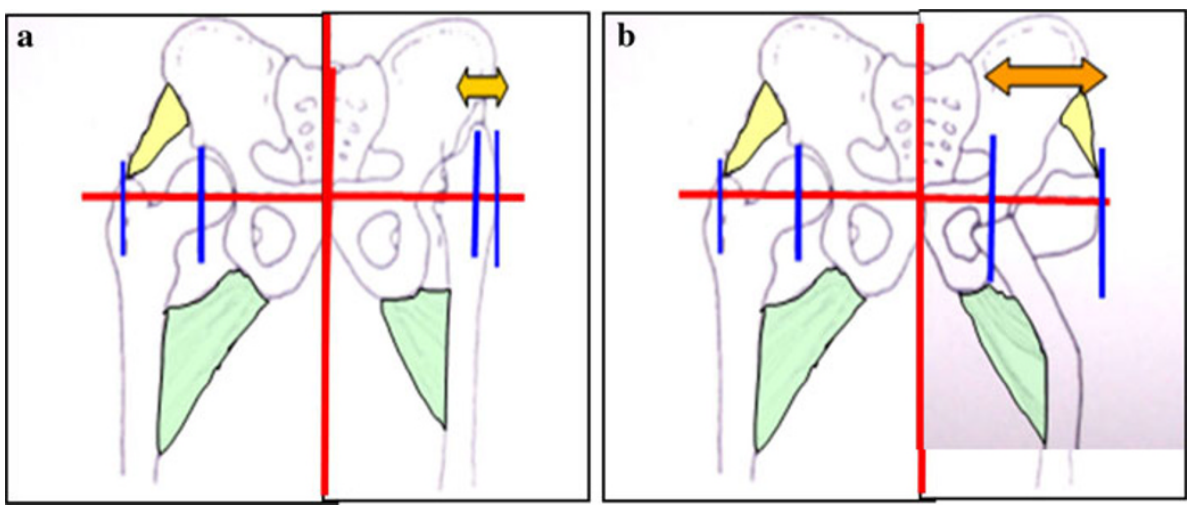

perform such operation. Ilizarov modified the concept of PSO by considering not only the frontal plane, but also the sagittal plane. He also added a distal femoral osteotomy to correct length discrepancy (LLD) and mechanical axis deviation $[2,11,12]$.

\section{Patients and methods}

Twenty hips in 20 patients were the candidates for PSO procedure. They were 5 men (25\%) and 15 women (75\%). The mean age was 21.5 years (range: 14-30). The etiology was neglected developmental dysplasia in 9 patients $(45 \%)$, post-septic hip sequelae in 9 patients $(45 \%)$, and paralytic post-polio dislocation in 2 patients $(10 \%)$. Nine patients had previous surgery. The two patients with poliomyelitis had multiple previous surgeries including soft tissue operations for hip flexion deformity, supracondylar extension osteotomy for knee flexion deformity, and foot triple fusion. Five patients of post-septic hip sequelae had previous surgical procedures; three of them had their hips drained through anterior approach, while the rest could not give a clear history about previous intervention. Two cases with dysplastic hips had previous failed open reductions through anterior approach.

\section{Preoperative evaluation}

All patients complained of limping and pain on ambulation. This was either thigh pain or low back pain. Patients were examined generally and locally with particular emphasis on Trendelenburg gait and sign, range of hip and knee motion, LLD, hip flexion deformity (by Thomas test) adduction and abduction, and spinal deformity.

The following radiographs are ordered: an anteroposterior radiograph of the pelvis showing both hips; an anteroposterior standing radiograph of both lower extremities, in neutral alignment with limb length equalized by blocks till a horizontal pelvic level is achieved, for performing Paley's malalignment test and measuring LLD (Fig. 2). Maximum adduction cross-legged anteroposterior
Fig. 2 Standing AP radiograph from pelvis to ankle for performing Paley's malalignment test and detecting the amount of LLD

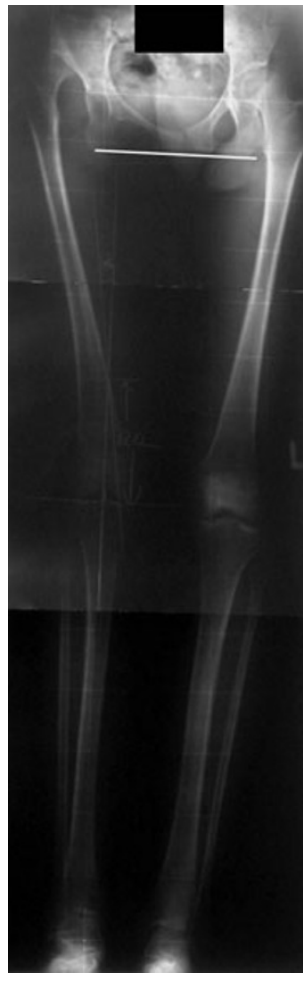

radiograph of the pelvis (made with the patient supine and the involved hip flexed and adducted over the thigh of the normal extremity) to determine the level and the angle of the proximal femoral osteotomy, where the femoral shaft radiologically approaches the ischium (taken as the most medial possible point to approach the pelvis) (Fig. 3).

The first osteotomy was chosen to be at the level where the femoral shaft radiologically approaches the ischium in the supine maximum adduction anteroposterior view. The valgus angle is made to be equal to the angle that the maximally adducted femur makes with the horizontal pelvic line (the maximum valgus angle that could be achieved). The degree of extension at the proximal osteotomy, needed to overcome the fixed hip flexion deformity and consequently to correct the hyperlordosis, was determined by exceeding the clinically premeasured hip flexion 


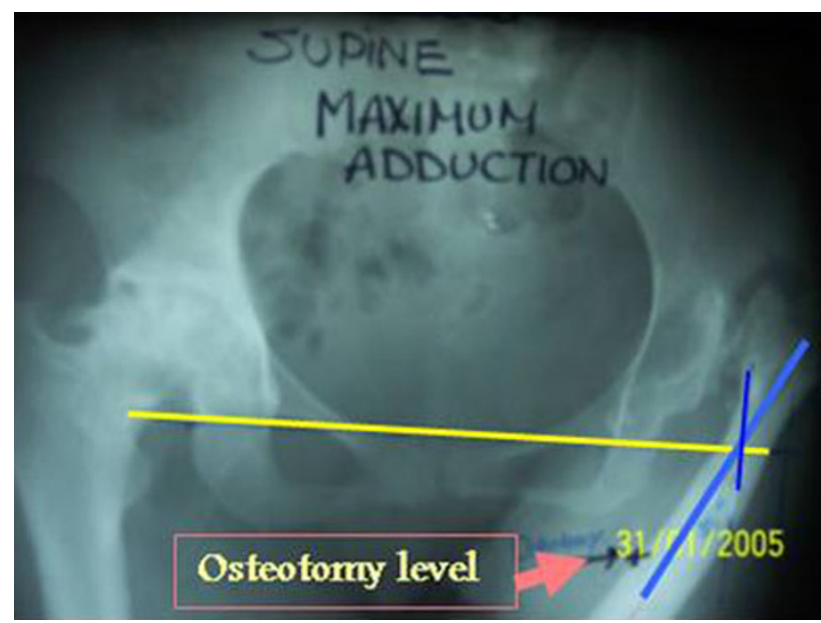

Fig. 3 Maximum adduction cross-legged supine radiograph

contracture, as recommended by Paley [12] and Rozbruch [3]. Those authors mentioned exactly $5^{\circ}$ in excess of the patient's flexion deformity, something we could not accurately reproduce intraoperatively (Fig. 4).

Goals of the second distal femoral osteotomy are to lengthen and realign the lower extremity. The level of the distal osteotomy is based on the amount of valgus at the proximal one; a paper tracing of the planned correction was performed in all cases. A line called the proximal mechanical axis line (PMA) was drawn perpendicular to the horizontal

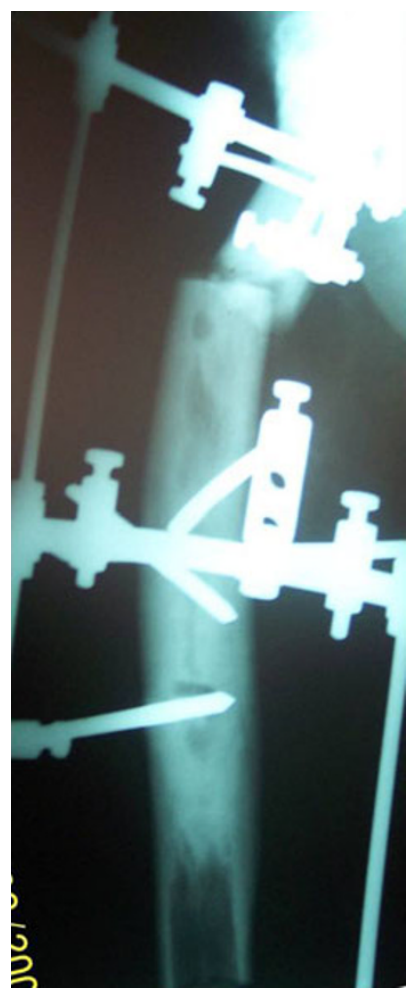

Fig. 4 Extension at the proximal osteotomy line of the pelvis passing through the region of the proximal osteotomy and extended distally. A distal line called the distal mechanical axis line (DMA) was drawn from the center of the ankle passing by the center of the knee and extended proximally. The intersection of the two lines marks the level of the second osteotomy (Fig. 5a, b). The level of the second osteotomy can be made slightly distal, but the distal femoral fragment will have to translate medially in addition to varus realignment. A more proximal location (diaphyseal) of the distal osteotomy is preferable in the authors' experience for two reasons; firstly, a longer distal fragment allows for adequate fixation away from the extensor expansion. Thus, more frame stability with earlier weight bearing, and better knee function. Secondly, a proximal location means less translation and consequently less expected frame adjustments during follow-up.

The magnitude of varus angulation at the distal osteotomy is equal to that of the angle between the DMA and PMA (Fig. 5a). Lengthening continues until the horizontal axis of the pelvis becomes parallel to the ground in standing radiographs.

The operative technique

The preassembled frame consists of two rings of appropriate diameter connected by a motor and two hinges.
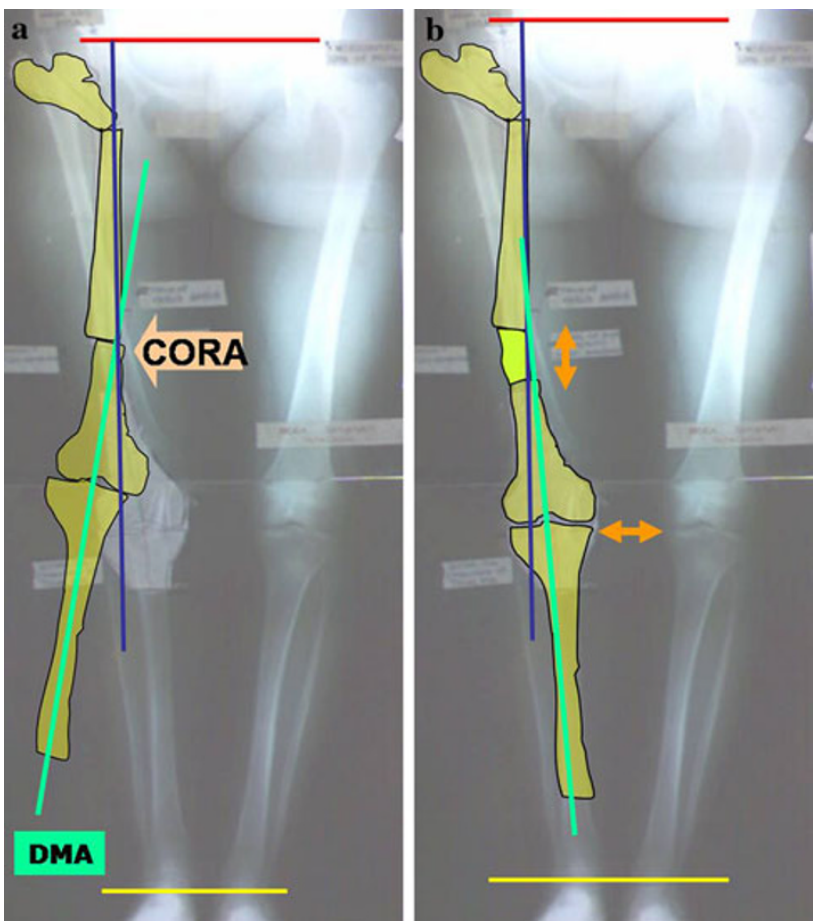

Fig. 5 a Paper tracing to plan for the value and level of distal osteotomy with the final situation imitated $(\mathbf{b})$. ( $P M A=$ proximal mechanical axis line, DMA = distal mechanical axis line, $C O R A=$ level of the second distal femoral osteotomy) 
Another modification is to connect both rings with four hinges mounted on the top of four rods that have quadrangular nuts in their distal connection. A $90^{\circ}$ arch is connected laterally to the proximal ring with two oblique supports. Lateral connection is important to avoid skin compromise at full assembly. Hinges are placed at the proximal ring whose level is adjusted to be at the planned level of the distal osteotomy. We mounted the distal frame with the rings either parallel or angulated by the predetermined angle of varization so that the final mounting ended (when the preplanned varus angulation achieved) with either a parallel or angulated position of the two rings consequently. Varization was done either acutely or gradually with lengthening.

Under general or regional anesthesia and after administration of parenteral antibiotics, the patient is placed on a radiolucent operating table with sheets placed under the sacrum to maintain a level pelvis and avoid rotation. With the use of image intensifier, a 1.8-mm Ilizarov wire, and a surgical marking pen, a line is marked across the inferior edge of the two sacroiliac joints; this is the horizontal pelvic line. Bony landmarks are also delineated. The predescribed assembly is fixed to the femur (with the middle ring at the level of distal osteotomy). A 6-mm threaded half pin is inserted from the posteromedial direction in the distal most ring. Another half pin is inserted from the posterolateral direction. This makes a stable distal delta construct (Fig. 6). More half pins are introduced to fix the proximal ring of the distal frame.

The assistant then holds the affected extremity maximally adducted and crossed over the uninvolved limb. This is done under image intensifier, and the femur is made to maximally approach the ischial tuberosity. This position forces the

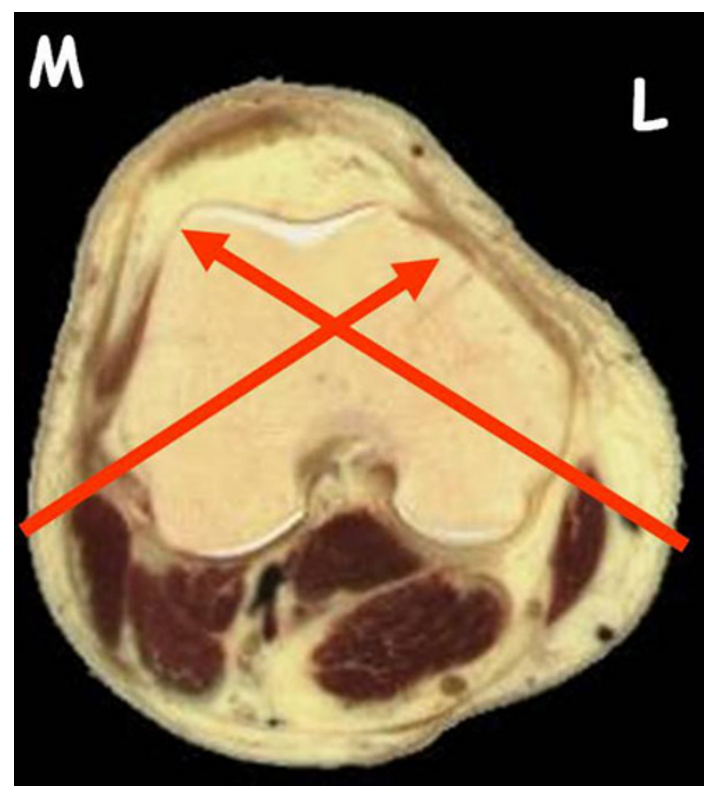

Fig. 6 Stable delta configuration of the most distal fixation femur to also rotate externally so as the proximal part of the femur has to confront to the plane of obturator foramen. A 6-mm threaded half pin is inserted parallel to the horizontal line of the pelvis and to the floor. The pin is connected to a free $90^{\circ}$ femoral arch. The arch is maintained perpendicular to the floor with the extremity crossed, automatically imparting extension at the proximal osteotomy, when made.

We find it difficult to produce an excess extension of exactly $5^{\circ}$ but rather we keep the amount that is automatically imparted when both limbs are brought parallel. More extension can be obtained during follow-up, to decrease the lumbar lordosis, by manipulating the arches.

A second 6-mm threaded half pin is then inserted anterolaterally into the femur distal to the predetermined osteotomy site and fixed to the arch attached to the distal frame, such that the angle between it and the first pin equals the predetermined valgus angle.

The first osteotomy is then made percutaneously through a 2- to 4-cm incision, and then the extremity is uncrossed till the two arches become parallel. We use a transverse incision because it is cosmetically favorable as edges coapt easily following angulation. The distal femoral fragment is rotated until the patella faces the ceiling.

The distal fragment is then displaced medially by manipulating arches before connecting them such that the lateral edge of the proximal most segment at the site of the osteotomy enters the medulla of the distal segment (this becomes the $\mathrm{m}$ middle segment after finishing both osteotomies). This, increases the stability of the proximal construct. The sequence of correction is rotation, translation, and angulation. The two arches are then connected with three threaded rods mounted with conical washers to adjust any malposition while still maintaining bony contact and correct angle at the proximal osteotomy.

The distal femoral osteotomy is then performed at the planned level. We routinely release the fascia and the lateral intermuscular septum from the same incision to neutralize their tethering effect during subsequent lengthening.

\section{Post-operative treatment}

Weight bearing with crutches can be allowed according to the individual case. Also passive and active range of motion of the knee and hip should be performed, complete weight bearing can be allowed after 3-4 weeks. Distraction starts 10 days after surgery at a rate of $1-\mathrm{mm} /$ day $(0.25 /$ $6 \mathrm{~h})$. This rate has to be slowed down in case of limitation of knee ROM (when a patient is not compliant with knee exercises) or if a patient experiences pin tract infection requiring a period of rest and antibiotic therapy. Lengthening was checked at each visit clinically using a measuring tap and at the end of distraction phase by a teleoroentgenogram. Varization in the distal osteotomy is 
done either acutely intraoperatively or gradually at the end of distraction phase through the laterally placed motor or by slowing the differential distraction at the 2 medial rods. Varization continues till DMA and PMA are aligned.

The frame is removed when three cortical bridging is detected on two X-ray views and after a period of dynamization (Fig. 7). The initial frame is cumbersome to patients especially with external fixation times extending up to 9 months. That is why we resort to a protocol of gradual frame disassembly;

- Removal of some of the most proximal pins (those fixing the proximal osteotomy) once it shows signs of satisfactory healing.

- Removal of the medial half of the proximal ring and adding more rods between its lateral half and the distal ring once the regenerate maturation is judged to be satisfactory. This leaves more space between the two thighs for more freedom during ambulation.

- Removal of the distal most wire again, once regenerated consolidation is near full to increase knee ROM before final frame removal.

- Removal of the most proximal arch when full removal is scheduled in the coming month or two.

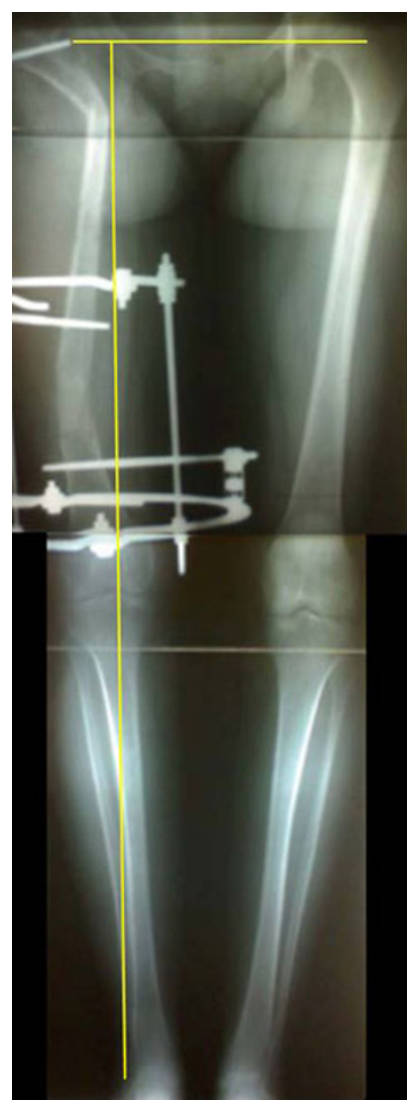

Fig. 7 A case during dynamization. The frame is left with 4 Schanz pins only

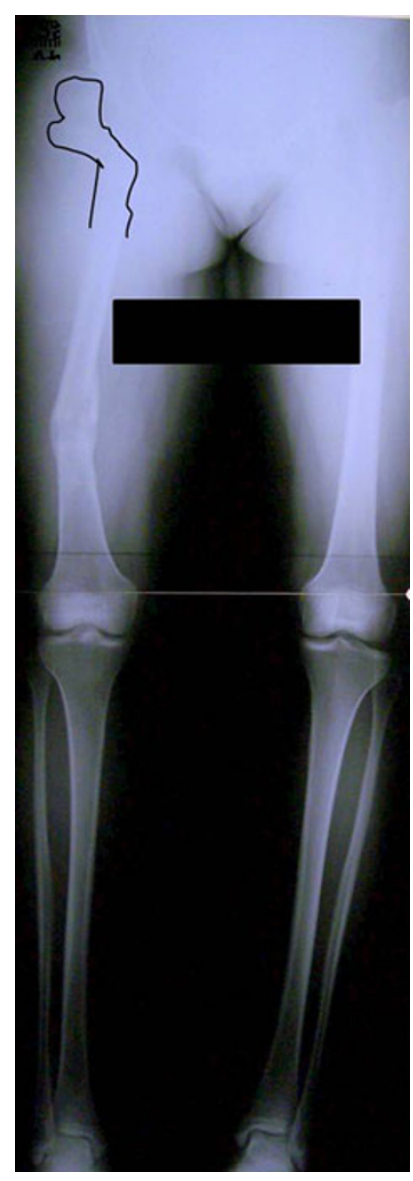

Fig. 8 Long film taken at final evaluation

Final clinical evaluation is done 6 months after frame removal (Fig. 8), and the results are classified into excellent, good, fair, and poor based on the following parameters: pain during walking and lying down, hip and knee range of motion, Trendelenburg sign, and the limblength discrepancy (Table 1).

\section{Results}

Subjective pain assessment for each case was done using the visual analog score (VAS) to compare between pain sensation pre- and post-operatively. Pain persisted in 13 of our patients but showed down scaling on the VAS compared with preoperative scale.

The mean external fixation time was 6.4 months (range, 5-9 months), and the mean preoperative limb-length discrepancy was $6.9 \mathrm{~cm}$ (range, 4-11 cm). Lengthening was achieved in all patients and the mean post-operative discrepancy became $1.1 \mathrm{~cm}$ (range, 0-3.5 cm). Mean mechanical axis deviation preoperatively was $13 \mathrm{~mm}$ in lateral direction (range, $15 \mathrm{~mm}$ in medial direction to 30-mm in lateral direction). Post-operatively, all limbs 
Table 1 Parameters used in evaluating the results of the patients

\begin{tabular}{|c|c|}
\hline $\begin{array}{l}\text { Result } \\
\text { category }\end{array}$ & Parameters \\
\hline $\begin{array}{l}\text { Excellent } \\
\text { result }\end{array}$ & $\begin{array}{l}\text { No pain ( } 0 \text { on VAS). } \\
\text { No LLD. } \\
\text { ROM equal to or better than before surgery. } \\
\text { Negative Trendelenburg sign }\end{array}$ \\
\hline Good result & $\begin{array}{l}\text { Mild pain }(0-3 \text { on VAS }) \\
\text { LLD }<2.5 \mathrm{~cm} . \\
\text { Reduced hip and/or knee ROM }<20^{\circ} \text {. } \\
\text { Negative or delayed Trendelenburg sign. }\end{array}$ \\
\hline Fair result & $\begin{array}{l}\text { Moderate pain ( } 4-6 \text { on VAS). } \\
\text { LLD }>2.5 \mathrm{~cm} . \\
\text { Reduced hip and/or knee ROM between } 20 \text { and } 30^{\circ} \text {. } \\
\text { Positive Trendelenburg sign. }\end{array}$ \\
\hline Poor result & $\begin{array}{l}\text { Continuous and/or sever pain (score } 7-10 \text { on VAS). } \\
\text { LLD }>5 \mathrm{~cm} \text {. } \\
\text { Reduced hip and/or knee ROM }>30^{\circ} \text {. } \\
\text { Positive Trendelenburg test. }\end{array}$ \\
\hline
\end{tabular}

were well aligned such that the mechanical axis crosses the middle of the tibial plateau (when PMA and DMA are aligned). The knee flexion range decreased from a mean of $138^{\circ}$ (range: $95-155^{\circ}$ ) preoperatively to a mean of $116.5^{\circ}$ (range, $60-135^{\circ}$ ) post-operatively. The decrease in knee flexion is explained by tightening of the quadriceps muscle associated with femoral lengthening. The mean preoperative hip range of flexion was $87.75^{\circ}$ (range, $40-130^{\circ}$ ), while post-operatively the mean was $75.25^{\circ}$ (range, $\left.30-120^{\circ}\right)$. The mean preoperative hip flexion contracture decreased from $16^{\circ}$ (range, $0-35$ ) to $3.25^{\circ}$ (range, $0-10^{\circ}$ ) post-operatively. The mean hip abduction increased from $37.75^{\circ}$ (range, $10-70$ ) to $45.75^{\circ}$ (range, $15-70^{\circ}$ ) postoperatively.

The decrease in hip flexion and increase in hip abduction were implemented by the direction of the proximal osteotomy. Trendelenburg sign was positive in all patients. It improved in 9 patients post-operatively, while disappeared in 11 patients (those patients where LLD was corrected to less than $2 \mathrm{~cm}$ and fixed hip flexion deformity was completely corrected). Trendelenburg sign was said to be improved when during the test it was initially absent but became positive after a while (due to earlier muscle fatigue and insufficient restoration of abductor power by the proximal osteotomy).

A scoring system was designed to evaluate the results based on the following parameters (Table 1):

- Pain by VAS

- LLD

- ROM of the hip and knee
- Trendelenburg test

Accordingly, we had 7 excellent results (35\%), 6 good results (30\%), 7 fair results (35\%), and no poor results.

\section{Complications}

All patients had pin tract infections that occurred mostly around the distal pins and during the distraction phase. Two cases had fracture regenerate that occurred within the first month after frame removal due to a fall. Both healed completely in a high above knee cast after 4 weeks. Premature consolidation occurred in 2 cases. Delayed consolidation was experienced in 4 patients, and this was treated during distraction phase by slowing the rate to $0.5 \mathrm{~mm} /$ day instead of $1 \mathrm{~mm} /$ day and at the end of distraction phase by callus message (compression-distraction at a rate $0.25 \mathrm{~mm} /$ day). No incidence of delayed healing of the proximal osteotomy was encountered. Residual limblength discrepancy of more than $2.5 \mathrm{~cm}$ required shoe lift in 3 patients to improve gait.

Most of the patients had knee stiffness immediately following frame removal due to prolonged external fixation time. We found that knee range of motion showed progressive improvement during the 6-month period following frame removal (Fig. 9), except in 2 cases: a male with poliomyelitis and a female with high dislocation who required $11 \mathrm{~cm}$ of lengthening. Both were non-compliant with physiotherapy.

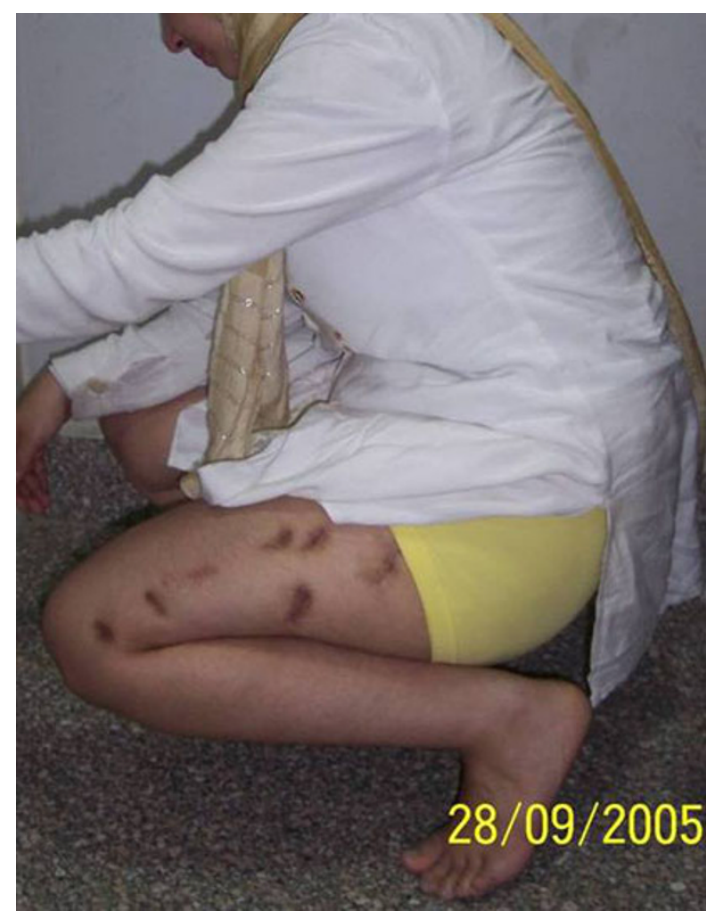

Fig. 9 Knee flexion at final evaluation 


\section{Discussion}

PSO for unstable hips has a long history in orthopedic surgery. With early procedures, increased stability was due to actual support of the pelvis on the osteotomized proximal part of the femur. In this type of reconstruction, the hip joint is not directly approached [5].

Hip arthroplasty, as an alternative option, has a high complication rate in young patients including peroneal nerve palsy, femoral nerve palsy, early post-operative dislocation, late infection, and aseptic loosening [13]. We agree with considerable amount of literature that total hip arthroplasty is best reserved for patients older than 40 years.

In this series, patients under 15 years of age were not candidates for the procedure. This is in agreement with the study by Milch [14], Gaenslen [15], Bombelli [5], and Kocaoglu et al. [4] who stated that PSO gives the best results in patients over 15 years of age otherwise the patient will have to repeat the procedure at or near skeletal maturity due to loss of proximal angulation with growth. In our study, the mean age was 21.5 years (range: 14-30). Adding this to the short-term follow-up, we did not experience or expect to experience loss of correction in any of our patients.

The optimal level for pelvic support has been controversial. Although some authors have recommended a proximal osteotomy with insertion of the lesser trochanter into the acetabulum, others have preferred a longer proximal segment. We are in favor of a more distal osteotomy, similar to that recommended by Schanz [15] or even lower because the more distal the osteotomy is made, the more medial the fulcrum is. This, increases the abductor force needed to balance the weight of the body in single-limb stance [15]. However, the pelvic abutment site is dictated by the patient's own anatomy as not every femur could be made to radiologically approach the ischial tuberosity at a fixed site in maximum adduction supine radiograph, i.e., the more the proximal migration, the lower the site of radiological pelvic contact and thus the implemented level of the proximal osteotomy.

Extension of the osteotomy contributes to hip stabilization in the sagittal plane. Thus, persistence of any flexion contracture eventually makes the pelvis unlocks itself from the "pelvic support" position and looses the fulcrum $[12,15]$. They are those patients whose flexion deformity could not be completely eliminated who showed unsatisfactory gait improvement.

We made the femur extend just more than the premeasured flexion deformity angle (by manipulating the arches through conical washers after both the limbs were made parallel). This was sufficient to lock the pelvis on standing. We could not make this angle exactly $5^{\circ}$ more than the flexion deformity angle as recommended by Paley [12] and Rozbruch et al. [3, 10]. In this series, the mean preoperative hip flexion contracture decreased from $16^{\circ}$ (range, 0-35) to $3.25^{\circ}$ (range, $0-10^{\circ}$ ) post-operatively.

Equalization of the lower-extremity length discrepancy is also important to improve gait mechanics. With LLD and without the use of a shoe lift, the pelvis is tilted. This alters the abductor lever arm and leaves room for adduction of the femur on the pelvis in single-limb stance. Therefore, without equalization of the lower-extremity length, pelvic drop cannot be prevented [5, 12]. None of our patients experienced knee subluxation during lengthening. This complication was even expected and prevented in the two poliomyelitis patients who showed knee instability during preoperative evaluation, by extending the frame to the tibia.

Trendelenburg gait is one of the hallmarks of unstable hips. With time, this is associated with increased pain due to muscle fatigue while walking, especially toward the end of the day. We found PSO to be very effective in eliminating the Trendelenburg gait and sign in these patients. No other method, except for arthrodesis, has been able to successfully address this aspect of the problem. In contrast to arthrodesis, it preserves an acceptable, painless range of motion of the hip. After PSO, ranges of hip flexion and adduction decrease while abduction and extension ranges increase. This is a kinematic fact dictated by the direction of the proximal angulation. In this work, the mean hip flexion range decreased by $25 \%$ and the mean abduction range increased by about $18 \%$. Rozbruch et al. [3] reported decrease in mean hip flexion of $26 \%$ and increase in hip abduction mean range by $20 \%$.

Knee stiffness is known to be caused by transfixion of muscles by Ilizarov wires and tightening of the quadriceps muscle associated with femoral lengthening procedures. In this work, knee range of motion was much limited in all patients immediately following fixator removal, but gradual improvement in knee flexion occurred during the following 6 months. Rozbruch et al. [3] reported mean loss of $9^{\circ}$ from the preoperative mean knee flexion range, after 60-month follow-up. Manzotti et al. [5] reported improved or unchanged knee range of motion in 8 of their 15 patients and loss of $10-20^{\circ}$ of the preoperative range in the rest after average follow-up of 108 months.

Kocaoglu et al. [4] reported loss of $24^{\circ}$ of mean knee flexion after average follow-up of 68 months. These results were also attributed to long follow-up period and good rehabilitation program in their institutions, to which the majority of patients were compliant (this was personally witnessed by the corresponding author who witnessed this during his fellowship in Istanbul University). The following tips are effective in preserving knee ROM during the fixation period: using a hybrid-advanced frame (pins 
completely replacing wires) $[2,5]$. In the middle femoral fragment, pins are better to be inserted from posterolateral direction. We found that three pins at three fixation levels in the middle fragment are enough for frame stability even with minimum angulation between them. Pins in the distal fragment are to be oriented $90^{\circ}$ to each other (from posteromedial and posterolateral directions). Pin insertion in the middle and distal femoral segments is done after adequate soft tissue releases with the knee flexed at $90^{\circ}$. While still under an anesthesia, after frame application and insertion of all pins, the knee is brought into full flexion range several tens of times to check for any limitation to full flexion. We believe that if the knee cannot be flexed fully intraoperatively, it will never do, but will worsen thereafter.

In our study, the limb is judged to be aligned when the proximal mechanical axis (PMA) and the distal mechanical axis (DMA) align such that the final mechanical axis passes by the tibial spine. Sometimes the CORA is too proximal that the distal osteotomy has to be made distal to it. Aligning the mechanical axes, hence, will need marked and impractical translation at the distal osteotomy. In these cases, alignment stops when both axes become parallel (limbs become parallel on standing films). We are worried about the long-term consequences of this on the knee joint as this definitely will increase the medial compartment stresses, an issue of unsolved debate.

In the final evaluation, the patient's results were categorized into four categories: excellent results in 7 patients, good results in 6 patients, fair results in 7 patients, and poor results in none of the patients. This evaluation system made us unable to compare our final results with results in literature, a draw back in our study. We should have used the modified Harris hip score to compare the functional outcome of PSO and total hip arthroplasty and to compare our functional results with the rest of literature. Further work is needed to evaluate the long-term changes that can occur at the frictional pelvic support site, and long-term follow-up is needed to see when patients will require conversion to arthroplasty. Comparative studies between PSO and hip arthroplasty in younger age population will present a definite answer to the research question.

Hip reconstruction by Ilizarov's technique can be technically demanding and involving lengthy period wearing the frame. However, it proved to be a valuable salvage procedure for numerous neglected hip problems particularly in young patients. Our results were encouraging, although long-term follow-up is needed.

Acknowledgments The corresponding author would like to express the deepest gratitude to Professor Mehmet Kocaoglu of Istanbul University, Turkey. He is a great professor, friend, and mentor.

Open Access This article is distributed under the terms of the Creative Commons Attribution License which permits any use, distribution and reproduction in any medium, provided the original author(s) and source are credited.

\section{References}

1. Callaghan JJ, Brand RA, Pedersen DR (1985) Hip arthrodesis. A long-term follow-up. J Bone Joint Surg Am 67(9):1328-1335

2. Maiocchi A (ed) (1994) Treatment of disorders of the hip. Special techniques. Il quadratino, Milan

3. Rozbruch S, Paley D, Behave A, Herzenberg J (2005) Ilizarov hip reconstruction for the late sequelae of infantile hip infection. J Bone Joint Surg Am 87A:1007-1018

4. Kocaoglu M, Eralp L, Sen C, Dincyurek H (2002) The Ilizarov hip reconstruction osteotomy for hip dislocation: outcome after 4-7 years in 14 young patients. Acta Orthop Scand 73(4):432-438

5. Bombelli R (ed) (1993) Structure and function in normal and abnormal hips: How To Rescue Mechanically Jeopardized Hips, 3th edn. Springer, Berlin

6. Manzotti A, Rovetta L, Pullen C (2003) Treatment of late sequelae of septic arthritis of the hip. Clin Orthop Relat Res 410:203-212

7. Stack JK, George K (1959) Pelvic support osteotomy for certain fracture complications of the hip. Am J Surg 97(5):644-648

8. Kirmission E (1894) De' l'osteotomie sous- trochante'rienne applique'e a certains cas de luxation conge'nitale de la hanche. (flexion de la cuisse avec adduction conside'rable). Rev d'Orthop 137

9. Shepherd MM (1960) A further review of the results of operations on the hip joint. J Bone Joint Surg Br 42B:177-204

10. Lorenz A (1919) Ueber die Behandlung der irreponiblen angeborenen Huftluxationen und der Schenkelhalspseudoarthrosen mittels Gabelung (Bifurkation des oberen Femurendes). Wien Klin Wchnschr. XXXII

11. Maiocchi A, Aronson J (eds) (1992) Treatment of disorders of the hip. Williams and Wilkins, Baltimore

12. Paley D (ed) (2002) Hip joint considerations. Springer, Berlin

13. MacKenzie JR, Kelley SS, Johnston RC (1996) Total hip replacement for coxarthrosis secondary to congenital dysplasia and dislocation of the hip Long-term results. J Bone Joint Surg Am 78(1):55-61

14. Milch H (1989) The "pelvic support" osteotomy. 1941. Clin Orthop Relat Res 249:4-11

15. Gaenslen F (1935) The schanz subtrochanteric osteotomy for irreducible dislocation of the hip. J Bone Joint Surg Am 17:76-78 